\title{
Efficient Parallel Tree Reductions on Distributed Memory Environments
}

\author{
Kazuhiko Kakehi $^{1}$, Kiminori Matsuzaki ${ }^{2}$, and Kento Emoto ${ }^{3}$ \\ ${ }^{1}$ Division of University Corporate Relations \\ ${ }^{2}$ Department of Mathematical Informatics \\ ${ }^{3}$ Department of Creative Informatics \\ University of Tokyo \\ $\{$ kaz, kmatsu, emoto\}eipl.t.u-tokyo.ac.jp
}

\begin{abstract}
A new approach for fast parallel reductions on trees over distributed memory environments is proposed. By employing serialized trees as the data representation, our algorithm has a communication-efficient BSP implementation regardless of the shapes of inputs. The prototype implementation supports its real efficacy.
\end{abstract}

Keywords: Tree reduction, parentheses matching, BSP, parallel algorithm.

\section{Introduction}

Research and development of parallelization have been intensively done toward matrices or one dimensional arrays. Looking at recent trends in applications, another data structure has also been calling for efficient parallel treatments: the tree structure. Emergence of XML as a universal data format, which takes the form of a tree, has magnified the impact of parallel and distributed mechanisms toward trees in order to reduce computation time and mitigate limitation of memory. However, parallel tree computation over distributed memory environments is not so straightforward as it looks.

Consider, as a simple and our running example, a computation maxPath to find the maximum of the values each of which is a sum of values in the nodes from the root to each leaf. When it is applied to the tree in Fig. 1, the result should be 12 contributed by the path of values $3,-5,6$ and 8 from the root. Parallelization of such a simple computation under distributed memory environments requires consideration from two aspects. The first is the underlying data representations, including its distribution among processors to guarantee performance toward trees of arbitrary shapes. The second is derivation of the parallel algorithm. As associativity often helps parallelization, we need to exploit the similar property under trees which suitably work for the data representations.

This paper gives a clear solution for parallel tree reductions with its start point to use serialized forms of trees. Their Notable examples are the serialized (streamed) representations of XML or parenthesized numeric expressions which are obtained by tree traversals. The first problem mentioned above is naturally resolved by this choice, since distribution of serialized data among processors is much simpler than that of trees. As for the second point, we present an efficient parallel algorithm for tree reductions satisfying extended distributivity. As instances of serialized trees, parallelization of parentheses matching problems, which figures out correspondence between brackets, have 


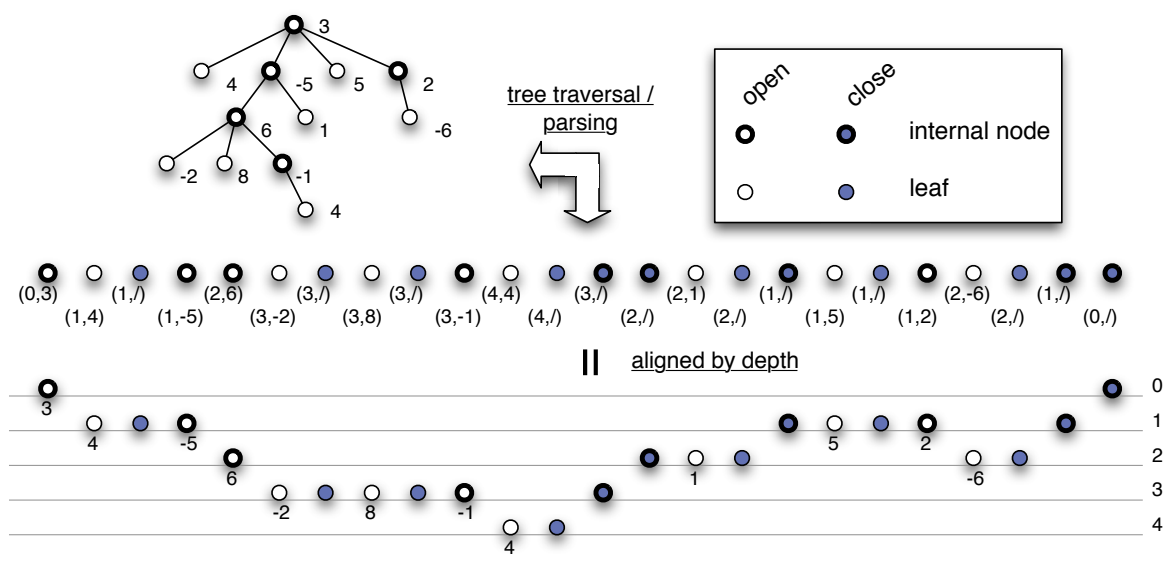

Fig. 1. A rose tree (upper left), its serialized representation as a sequence of pairs (middle) and another representation according to the depth (lower)

plenty of work ( [1, 2, 3, 4, 5] to mention a few); our algorithm, with good resemblance to the one under BSP [6], has also a BSP implementation with three supersteps.

This paper is organized as follows. After this Introduction, Sect. 2 observes our tree representations and tree reductions. Section 3 develops the algorithm. Our algorithm consists of three phases, where the first two perform computation along with parentheses matching, and the last reduces a tree of size less than twice of the number of processors. Section 4 supports our claims by some experiments. We conclude this paper in Sect. 5] and mentioning future directions. We refer the interested reader to our technical report [7] for the omitted details due to the space limitation of this paper.

\section{Preliminaries}

This section observes the underlying frameworks for our parallelization: tree structures, their serialized form, tree homomorphism, and extended distributivity.

\subsection{Trees and Their Serialized Representation}

We treat trees with unbound degree (trees whose nodes can have an arbitrary number of subtrees); Fig. 1 shows an example. As was explained in Introduction, our internal representation is to keep tree-structured data in a serialized manner. The sequence of the middle in Fig. 1 is our internal representation of the example tree. Like XML serialization or parentheses expressions, it is a combination of a preorder (for producing the open elements) and a postorder traversal (for producing the close elements afterwards). We assume well-formedness, with which sequences are guaranteed to be parsed back into trees, and without loss of information we simplify close elements to be "/". For the convenience of later discussion, we assign the information of the depth in the tree to each node. The figure also depicts their presentation according to the depth. 


\subsection{Tree Homomorphism and Extended Distributivity}

We use the framework called tree homomorphism [8], which specifies recursive tree reductions $h$ using $h^{\prime}, \oplus$, and associative $\otimes$ with its unit $\iota_{\otimes}$ :

$$
\begin{aligned}
h\left(\text { Node } a\left[t_{1}, \ldots, t_{n}\right]\right) & =a \oplus\left(h\left(t_{1}\right) \otimes \cdots \otimes h\left(t_{n}\right)\right), \\
h(\text { Leaf } a) & =h^{\prime}(a) .
\end{aligned}
$$

The computation maxPath mentioned in Introduction is also a tree homomorphism:

$$
\begin{aligned}
\operatorname{maxPath}\left(\text { Node } a\left[t_{1}, \ldots, t_{n}\right]\right) & =a+\left(\operatorname{maxPath}\left(t_{1}\right) \uparrow \cdots \uparrow \operatorname{maxPath}\left(t_{n}\right)\right), \\
\operatorname{maxPath}(\text { Leaf } a) & =i d(a)=a .
\end{aligned}
$$

Here, $i d$ is the identify function, and $\uparrow$ returns the bigger of two numbers whose unit is $-\infty$. When it is applied to the tree in Fig. 1 the result should be $12=3+(-5)+6+8$.

We can apply parallelization of tree homomorphism over serialized trees based on list homomorphism [3]. This naive approach, however, suffers from the factor of tree depths. We need to review an additional property called extended distributivity [9]. This property is explained, by introducing a new operator $\ominus$ defined as $(a, b, c) \ominus e=a \oplus$ $(b \otimes e \otimes c)$, as follows: for any triples $\left(a_{u}, b_{u}, c_{u}\right),\left(a_{l}, b_{l}, c_{l}\right)$, and any expression $e$, there exists a triple $(a, b, c)$ which satisfies

$$
\left(a_{u}, b_{u}, c_{u}\right) \ominus\left(\left(a_{l}, b_{l}, c_{l}\right) \ominus e\right)=(a, b, c) \ominus e .
$$

Efficient parallel reduction requires these computations as well as $\otimes$ and $\oplus$ to be done in constant time. Our running example satisfies these properties as the following calculation shows.

$$
\begin{aligned}
& \left(a_{u}, b_{u}, c_{u}\right) \ominus\left(\left(a_{l}, b_{l}, c_{l}\right) \ominus e\right) \\
& =a_{u}+\left(b_{u} \uparrow\left(a_{l}+\left(b_{l} \uparrow e \uparrow c_{l}\right)\right) \uparrow c_{u}\right) \\
& =\left(a_{u}+a_{l}\right)+\left(\left(-a_{l}+b_{u} \uparrow b_{l}\right) \uparrow e \uparrow\left(c_{l} \uparrow-a_{l}+c_{u}\right)\right) \\
& =\left(\left(a_{u}+a_{l}\right),\left(-a_{l}+b_{u} \uparrow b_{l}\right),\left(c_{l} \uparrow-a_{l}+c_{u}\right)\right) \ominus e
\end{aligned}
$$

For some other examples under these formalizations, see our previous work [9].

\section{Parallel Computation over the Serialized Trees}

This section develops a parallel algorithm for tree homomorphism which satisfies extended distributivity. We explain our algorithm in three phases: (1) the first phase applies tree homomorphism toward segments (consecutive subsequences) in each processor as much as possible; (2) after communications among processors the second phase performs further reduction using extended distributivity, producing a binary tree as a result whose internal nodes are specified as triples, and size is less than twice of the number of processors; finally (3) the third phase reduces the binary tree into a single value.

We use $\mathrm{N}$ and $\mathrm{P}$ to the number of tree nodes and available processors, respectively. During the explanation we assume $\mathrm{P}=4$. The algorithm resembles the analysis of parentheses matching problems under BSP [5].

First phase. Each processor applies tree homomorphism to its given segment of size $2 \mathrm{~N} / \mathrm{P}$. The process is summarized as Routine 1 . This process leaves fragments of results, 


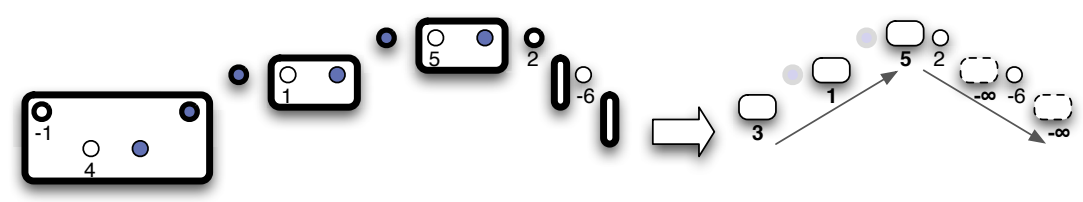

Fig. 2. An illustrating example of the first phase-applying tree homomorphism maxPath to a segment of Fig.1 (lined and dashed ovals indicate values obtained by reduction and $-\infty$ (the unit of $\uparrow$ ), respectively)

in arrays $a s_{p}, b s_{p}, c s_{p}$ and an integer $d_{p}$ for each processor number $p$. The array $a s_{p}$ is to keep the open elements without their corresponding close element. Each element of $a s_{p}$ can have subtrees before the next one in $a s_{p}$, and their reduced values are kept in $b s_{p}$. Similar treatments are done to unmatched close elements, leaving values in $c s_{p}$ (we remove unmatched close elements thanks to the absence of values). The integer $d_{p}$ denotes the shallowest depth in processor $p$. While both of elements in $a s_{p}$ and $b s_{p}$ are listed in a descending manner, those of $c s_{p}$ are in ascending manner; the initial elements of $a s_{p}$ and $b s_{p}$ and the last one of $c s_{p}$ are at height $d_{p}$ (except for $c s_{0}$ and $c s_{\mathrm{P}_{-1}}$ whose last element at depth 0 is always $\iota_{\otimes}$ and therefore is set to be eliminated).

Routine 1. Prepare arrays as, bs, cs (behaving as stacks growing from left to right), and an integer variable $d$. A temporary $t$ is used. Sequence $\left(d_{0}, a_{0}\right), \ldots,\left(d_{n-1}, a_{n-1}\right)$ $(n \geq 1)$ is given.

- Set $d \leftarrow d_{0}$. If $a_{0}$ is “/" then $c s \leftarrow\left[\iota_{\otimes}, \iota_{\otimes}\right]$, as $\leftarrow[], b s \leftarrow[]$; else $c s \leftarrow\left[\iota_{\otimes}\right]$, $a s \leftarrow\left[a_{0}\right], b s \leftarrow\left[\iota_{\otimes}\right]$.

- For each $i$ in $\{1, \ldots, n-1\}$

- if $a_{i}$ is not "/" (namely a value), then push $a_{i}$ to as and $\iota_{\otimes}$ to $b s$;

else $\bullet$ if as is empty, then push $\iota_{\otimes}$ to $c s$, and set $d \leftarrow d_{i}$;

else $\bullet$ pop $a^{\prime}$ from $a s$ and $b^{\prime}$ from $b s$.

- if $b^{\prime}=\iota_{\otimes}$ (implying $a^{\prime}$ is a leaf) then $t \leftarrow h^{\prime}\left(a^{\prime}\right)$; else $t \leftarrow a^{\prime} \otimes b^{\prime}$;

- if $b s$ is not empty, then pop $b^{\prime \prime}$ from $b s$ and push $b^{\prime \prime} \otimes t$ to $b s$; else pop $c^{\prime \prime}$ from $c s$ and push $c^{\prime \prime} \otimes t$ to $c s$.

- If $\mathrm{P} \neq 1$ then remove $\iota_{\otimes}$ at the bottom of $c s_{0}$ and $c s_{\mathrm{P}-1}$.

In Fig. 2 we show a case of the illustrating segment from the 10th element $(3,-1)$ to the 21 st $(2,-6)$ of the sequence in Fig. 1. We have, as depicted:

$$
\begin{aligned}
c s & =[-1+i d(4), i d(1), i d(5)] & & \text { as }=[2,6], \\
& =[3,1,5], & & b s=[-\infty,-\infty],
\end{aligned}
$$

Please note that we regard absence of subtrees as an empty forest to which the tree homomorphism returns $-\infty$, the unit of $\uparrow$ (at depth 2 and 3 kept in $b s$ ). When we distribute the whole sequence in Fig. 1 evenly among four processors ( 6 elements for each), the results by this phase is shown in Fig. 3. left.

A linear routine produces $a s, b s, c s$ and $d$. The worst case in terms of the size of the results occurs when an sequence of only open elements is given, resulting in two arrays as and $b s$ of the length $2 \mathrm{~N} / \mathrm{P}$ for each. 

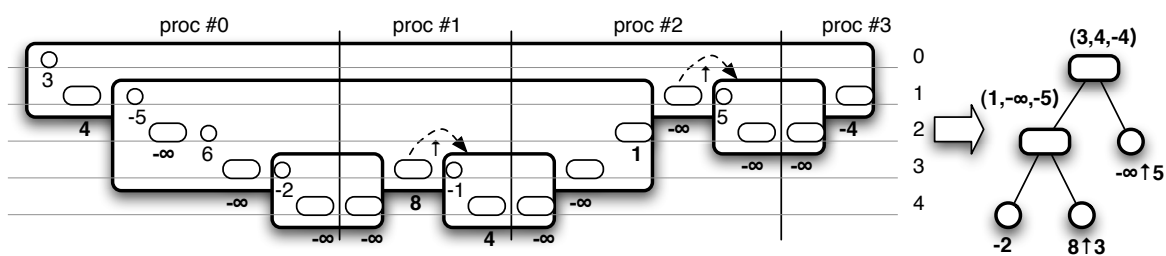

\begin{tabular}{|c|c|c|c|}
\hline$c s_{0}=[]$ & $c s_{1}=[-\infty, 8]$ & $c s_{2}=[-\infty,-\infty, 1,-\infty]$ & $c s_{3}=[-\infty,-4]$ \\
\hline$a s_{0}=[3,-5,6,2]$ & $a s_{1}=[-1]$ & $a s_{2}=[5]$ & $a s_{3}=$ \\
\hline$b s_{0}=[4,-\infty,-\infty,-\infty]$ & $b s_{1}=[4]$ & $b s_{2}=[-\infty]$ & $b s_{2}=[]$ \\
\hline$d_{0}=0$ & $d_{1}=3$ & $d_{2}=1$ & $d_{3}=0$ \\
\hline
\end{tabular}

Fig. 3. Triples between two processors (left) and the resulting tree (right) after the second phase

Second phase. The second phase matches data fragments kept in each processor into triples using communication between processors. Later, we reduce consecutive occurrences of triples into a value, or into one triple by extended distributivity.

When we look carefully at Fig. 3. we notice that 3 in $a s_{0}$ at depth 0 now has five parts at depth 1 as its children: the value 4 in $b s_{0}$, a subtree spanning from processors 0 to 2 whose root is -5 in $a s_{0}$, the value $-\infty$ in $c s_{2}$, a subtree from processor 2 to 3 whose root is 5 in $a s_{2}$, and the value -4 in $c s_{3}$. As these subtrees need reducing separately, we focus on the leftmost and the rightmost values in $b s_{0}$ and $c s_{3}$ (we leave the value $-\infty$ in $c s_{2}$ for the time being). We notice that the group of the value 3 in $a s_{0}$ with these two values in processors 0 and 3 forms a triple $(3,4,-4)$.

Similarly, two elements in $a s_{0}$ at depth 1 and 2, with two elements each in $b s_{0}$ and $c s_{2}$ at depth 2 and 3 , respectively, form two triples $(-5,-\infty, 1)$ and $(6,-\infty,-\infty)$. The former triple indicates a tree that awaits the result of one subtree specified by the latter. This situation is what extended distributivity takes care of: we can merge two triples (a sequence of triples in general) into one: $(-5,-\infty, 1) \ominus((6,-\infty,-\infty) \ominus e)=$ $((-5+6),(-6-\infty \uparrow-\infty),(-\infty \uparrow-6+1)) \ominus e=(1,-\infty,-5) \ominus e$ for any $e$. In this way, the group of data fragments in two processors turn into one triple.

Such groups from two adjacent processors are reduced into a single value without any missing subtrees in between: instead of treating using extended distributivity, the values -2 in $a s_{0}$ and -1 in $a s_{1}$ at depth 3, and 5 in $a s_{2}$ at depth 2 with their corresponding values in $b s_{i}$ and $c s_{i+1}(i=0,1,2)$ turn into values $i d(-2)=-2$, $-1+(4 \uparrow-\infty)=3, i d(5)=5$, respectively.

We state the following lemma to tell the number of resulting groups in total.

Lemma 1. The second phase produces groups of the number at most $2 \mathrm{P}-3$.

The proof sketch is as follows. Let $R_{p}$ be the the number of groups among $p$ processors. As Fig. 3 indicates, $R_{2}=1$, and for $p>2$ we derive $R_{p} \leq 1+R_{j}+R_{p-j+1}$ with $1<j<p$; hence we have $R_{p} \leq 2 p-3$. This lemma guarantees that, after transactions of data fragments among processors, one processor needs to take care of at most two such groups. The computed value at the shallowest depth $d_{p}$ in each processor are associated to their right for later computation by $\uparrow$ ( 8 in $c s_{1},-\infty$ in $c s_{2}$; see Fig. 3).

The following Routine 2 figures out groups among processors. $M_{\left[d_{u}, d_{l}\right]}^{p_{l} \leftrightarrow p_{r}}$ denotes a group between processors $p_{l}$ and $p_{r}$ whose data fragments span from the depth $d_{u}$ until $d_{l}\left(\infty\right.$ in $d_{l}$ indicates "everything starting from $d_{u}$ "); $M_{\left[-\overleftarrow{d}_{u}, \bar{d}_{l}\right]}$ is inserted as a dummy 
group in case the same $d$ appear among more than two consecutive processors, and assumed to be reduced into $\iota_{\ominus}$, a virtual left unit of $\ominus$ (namely $\iota_{\ominus} \ominus e=e$ ).

Routine 2. A stack is used whose top is referred as $\left(p_{s}, d_{s}\right)$. Sequence $\left(p, d_{p}\right)$ is given in the ascending order of $p$.

- Push the first pair $(0,0)$ on a stack

- For each $i$ in $\{1, \ldots, \mathrm{P}-1\}$

- prepare a variable $d \leftarrow \infty$.

- while $d_{i}<d_{s}$, produce $M_{\left[d_{s}, d\right]}^{p_{s} \leftrightarrow i}$, set $d \leftarrow d_{s}$ and pop from the stack;

- if $d_{i}=d_{s}$, then produce $M_{\left[d_{s}, d\right]}^{p_{s} \leftrightarrow i}$ and $M_{\left[\bar{d}_{i}, \bar{d}_{s}\right]}$, and pop from the stack. else produce $M_{\left[d_{i}, d\right]}^{p_{s} \leftrightarrow i}$.

- $\operatorname{push}\left(i, d_{i}\right)$.

- Finally eliminate the last mating pair (that is $M_{[0,0]}^{-}$).

We summarize this phase. This phase consists of two steps. In the first all processors figure out the groups by Routine 2 and allocation of groups by any deterministic rule through sharing their depth information $d_{i}$. They require $\mathrm{O}(\mathrm{P})$ communication and computation costs in total. The second step is to apply further computation toward each group. In the worst case it is possible that a processor sends out its whole data fragments in it to all other $\mathrm{P}-1$ processors, and receives two groups and evaluates each into one triple or value. Since the size of fragments in one processor and that of each group are at most $\mathrm{O}(\mathrm{N} / \mathrm{P})$, the cost for data transaction and computation is bound by $\mathrm{O}(\mathrm{N} / \mathrm{P})$.

Third phase. This last phase compiles obtained triples or values and reduce them into a single value. As Fig. 3 shows, the obtained triples and values in the previous phase form a binary tree of size $2 \mathrm{P}-3$ (including dummies by $M_{[0,0]}$ ). We collect triples or values in one processor, and apply tree reduction in $\mathrm{O}(\mathrm{P})$ time.

In summary, we have three communication rounds (two in the second phase, one in the third phase). We conclude this section by stating the following theorem.

Theorem 1. Tree homomorphism with extended distributivity has a BSP implementation with three supersteps of at most $\mathrm{O}(\mathrm{P}+\mathrm{N} / \mathrm{P})$ communication cost for each.

\section{Experiments and Discussion}

We performed experiments using our prototype implementation using $\mathrm{C}++$ and MPI. Specifications of our PC cluster are shown in Table 1, left. We simulated a simple query on rose trees where local computation of $\otimes$ and $\oplus$ were matrix multiplication-like operations over matrices of the size $10 \times 10$. We prepared randomly generated trees of three types, namely (RS) shallow, (RD) deep, and (M) a monadic tree (a tree-view of a list). The tree size was constrained by the memory size a machine has. The height of RS came from observations on XML documents [10]. We executed the program over each type using $2^{i}$ processors $(i=0, \ldots, 6)$.

Table 1 summarizes the results. First, it is natural that no difference existed in terms of costs of initial data distribution. As their plots in the left of Fig. 4 indicates, our algorithm exhibited good scalability for both of (RS) and (RD). Results of (M) fell 
Table 1. Specification (left) and execution times (right) of our experiments

\begin{tabular}{l|l}
\hline \hline machine & $2.8 \mathrm{GHz}$ CPU, 2GB memory \\
\hline network & Gigabit Ethernet \\
\hline software & Linux 2.4.21, \\
& gcc 2.96, mpich 1.2.7 \\
\hline \hline data: randomly generated trees of size \\
2,000,000 \\
(RS) with maximum height 7 \\
(RD) with maximum height of 5,000 \\
(M) a monadic tree \\
\hline
\end{tabular}

\begin{tabular}{r|r||r|c||c|c||c|c}
\hline \hline & \multicolumn{1}{|c||}{} & \multicolumn{2}{c||}{ Deep (RD) } & \multicolumn{2}{c||}{ Shallow (RS) } & \multicolumn{2}{c}{ Monadic (M) } \\
\cline { 3 - 8 } $\mathrm{P}$ & dist. & comp. & total & comp. & total & comp. & total \\
\hline 1 & - & 23.5 & 23.5 & 22.8 & 22.8 & N.A. & N.A. \\
2 & 0.48 & 12.3 & 12.7 & 12.6 & 13.0 & N.A. & N.A. \\
4 & 0.56 & 6.13 & 6.70 & 5.81 & 6.36 & 60.1 & 60.7 \\
8 & 0.62 & 3.18 & 3.80 & 3.79 & 4.41 & 20.9 & 21.5 \\
16 & 0.70 & 1.81 & 2.51 & 1.96 & 2.65 & 14.8 & 15.5 \\
32 & 0.77 & 1.11 & 1.88 & 1.04 & 1.81 & 8.36 & 9.14 \\
64 & 0.83 & 0.68 & 1.52 & 0.57 & 1.40 & 4.47 & 5.30 \\
\hline
\end{tabular}
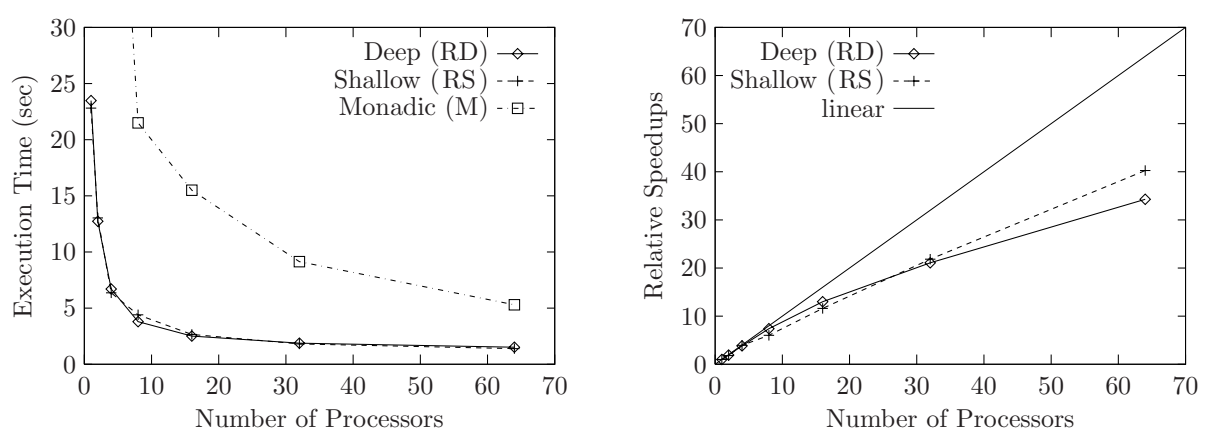

Fig. 4. Plots of Table 1 by total execution time (left) and by speedups of computation time (right)

behind the other two: it ran out of memory until 2 processors (the result by 4 processors seems also affected), and its execution was around seven times slower than other cases. The reason is that there are no reducible subtrees in the first phase, which incurs heavy data transactions and costly computation by extended distributivity. It should be noted that the similar BSP algorithm for all nearest smaller values problem, generalization of parentheses matching, toward average cases is shown not to suffer from heavy transactions [5]. While we need to develop a similar theoretical proof to our algorithm, experiments on random trees (RS and RD) suggest that our algorithm works efficiently toward average cases.

We make a brief comparison with existing approaches of parallel tree computations. One common approach under distributed memory environments has their basis in list ranking, and their parallel tree contractions require expensive costs of $\mathrm{O}(\mathrm{N} / \mathrm{P} \log \mathrm{P})$ [11, 12, 13]. Using a technique called $m$-bridge [14] for initial data distribution, our existing library implements parallel tree contractions in $\mathrm{O}(\mathrm{N} / \mathrm{P}+\mathrm{P})$ over trees kept as linked structures [15, 16, 9]. In comparison with them, the approach in this paper has two notable advantages. First, it can be coded as simple for-loops over one-dimensional arrays and benefits compiler optimizations well. We also observed that high memory locality by serialized forms brought considerable performance improvements, especially when the required computation was memory-intensive where cache effects become important. Second, the data distribution process in this paper is really small compared to the cost of $m$-bridge, in which traversal over linked structure is involved. 


\section{Concluding Remarks}

In this paper we have developed a new approach to reduce a tree in parallel. The algorithm has been shown to run scalably as well as fast in theory and practice, by exploiting the serialized trees and a property called extended distributivity.

At the moment we have only analyzed cost for the worst case. It is our interesting task to analyze average cases, or go a step further to the theory of heterogeneous cases.

Acknowledgment. This research was partially supported by the Ministry of Education, Culture, Sports, Science and Technology, Grant-in-Aid for Young Scientists (B), 17700026, 2005-2007.

\section{References}

1. Berkman, O., Schieber, B., Vishkin, U.: Optimal doubly logarithmic parallel algorithms based on finding all nearest smaller values. Journal of Algorithms 14 (1993)

2. Prasad, S., Das, S., Chen, C.: Efficient EREW PRAM algorithms for parentheses-matching. IEEE Transactions on Parallel and Distributed Systems 5(9) (1994)

3. Cole, M.: Parallel programming with list homomorphisms. Parallel Processing Letters 5 (1995)

4. Kravets, D., Plaxton, C.: All nearest smaller values on the hypercube. IEEE Transactions on Parallel and Distributed Systems 7(5) (1996)

5. He, X., Huang, C.: Communication efficient BSP algorithm for all nearest smaller values problem. Journal of Parallel and Distributed Computing 16 (2001)

6. Valiant, L.: A bridging model for parallel computation. Communication of the ACM 33(8) (1990)

7. Kakehi, K., Matsuzaki, K., Emoto, K., Hu, Z.: A practicable framework for tree reduction under distributed memory environments. Technical Report METR 2006-64, Department of Mathematical Informatics, University of Tokyo (2006)

8. Skillicorn, D.B.: Parallel implementation of tree skeletons. Journal of Parallel and Distributed Computing 39(2) (1996)

9. Matsuzaki, K., Hu, Z., Kakehi, K., Takeichi, M.: Systematic derivation of tree contraction algorithms. Parallel Processing Letters 15(3) (2005) (Original version appeared in Proc. 4th International Workshop on Constructive Methodology of Parallel Programming, 2004.).

10. Mignet, L., Barbosa, D., Veltri, P.: The XML web: a fist study. In Proceedings of the Twelfth International World Wide Web Conference, ACM Press (2003)

11. Mayr, E.W., Werchner, R.: Optimal routing of parentheses on the hypercube. Journal of Parallel and Distributed Computing 26(2) (1995)

12. Mayr, E.W., Werchner, R.: Optimal tree contraction and term matching on the hypercube and related networks. Algorithmica 18(3) (1997)

13. Dehne, F., Ferreira, A., Cáceres, E., Song, S., Roncato, A.: Efficient parallel graph algorithms for coarse-grained multicomputers and BSP. Algorithmica 33(2) (2002)

14. Reid-Miller, M., Miller, G.L., Modugno, F.: List ranking and parallel tree contraction. In Reif, J.H., ed.: Synthesis of Parallel Algorithms. Morgan Kaufmann (1993)

15. SkeTo Project Home Page. http: / /www . ipl.t.u-tokyo.ac.jp/sketo/

16. Matsuzaki, K., Emoto, K., Iwasaki, H., Hu, Z.: A library of constructive skeletons for sequential style of parallel programming (invited paper). In: Proceedings of the First International Conference on Scalable Information Systems, IEEE Press (2006) 